\title{
AN INTERACTIVE APPLICATION TO ASSIST BIOLOGY LEARNING USING AUGMENTED-REALITY
}

\author{
Wenxi $\mathrm{Li}^{1}$, Marisabel Chang ${ }^{2}$, Yu Sun ${ }^{2}$ \\ ${ }^{1}$ Northwood High School New City, Irvine, CA 92620 \\ ${ }^{2}$ Department of Computer Science, California State Polytechnic University, \\ Pomona, USA
}

\begin{abstract}
As students learn biology in term of molecule, cells, or proteins, cross section of $2 D$ image is a traditional method study the details of them. However, this method cannot bridge the gap between reality and students' imagination based on 2D image. This paper proposes a tool which can assist students to learn biology knowledge more effectively through augmented-reality. The experiments on accuracy and performance of the image pattern recognition indicates that FAST algorithm is the best choice currently. It reaches the highest accuracy of $94.5 \%$.
\end{abstract}

\section{KEYWORDS}

Augment reality, Vuforia, Blender, Unity

\section{INTRODUCTION}

When coming to a biology molecule-for example, cells and proteins-it can be quite hard for students to visualize. Traditionally, students can see cross sections of the molecule in 2D images. However, there is always a gap between the sides to sides as well as the reality's conception and the audience's imagination. For example, the overlapping sections in tissues would be hard to recognize when given a cross-section. The emergence of 3D digitalization has altered such scenarios profoundly. When applying 3D modeling to biological molecules, instructors may describe and convey the knowledge more vividly and students may understand the designs more fully and more accurately in a more convenient way.

Open Problem: Although students can use diverse methods to learn biological molecule such as 3D digital and 3D physical models, they are no efficiency since they are costly, complex and inaccessible. Some digital 3D modeling techniques and systems that have been proposed to demonstrate biology structures include websites like sketchfab and Turbosquid [12][13]. Sketchfab and Turbosquid are platforms that sell, publish, share, and buy 3D model content. However, these websites are constructed as data bases for 3D models and their goal is to establish a platform for people to post and trade their 3D models. These platforms do not have enough biology models specifically made for school purposes, therefore, a lot of the biology models they have are either too abstract or complex for student understanding. Besides digital 3D modeling, using physical 3D biology molecule models for demonstration is another option. However, physical 3D models are relatively inefficient, considering making a physical model is usually time consuming and limited people can have access to the model [20]. Nevertheless, 
physical models are usually rigid, unlike digital models which students can zoom in and out and view the interior of the models.

Solution: built a 3D biological molecules model using Augmented Reality and Computer Vision. Software's or websites specifically dedicated to demonstration of 3D biological molecules is a relatively innovative and unique idea. When coming to the purpose of teaching and demonstration to facilitate student understanding, a software specifically dedicated to biology models is especially practical, efficient, and engaging, since it can be downloaded on digital devices easily and gives students a thorough understanding of the biology molecule.

For our mobile application, we used Unity3D, Blender and Vuforia. Unity3D is the game engine we used to import the 3D biological molecule models and 3D target model to create the mobile application. We chose Unity3D since it supports cross platform development for both android and IOS. Blender is an open-source 3D modeling software tool that we used to create some of the 3D biological molecule models.[5][7] Vuforia is an Augmented-Reality software for mobile application developed by Qualcomm [14]. It uses computer vision technology to track and recognize image in real time [4]. I used it to assist recognition of the images.

Two experiments have been conducted to verify the following two aspects of the system:

Experiment 1: The accuracy of the image pattern recognition using different algorithms. The core image pattern recognition algorithms rely on the image feature extraction methods. We have tested the different feature extraction algorithms to recognize the image pattern based on the chosen sampled test images, such as FAST, Difference of Gaussians, and Determinant of Hessian. It turns out that FAST has the highest accuracy of $94.5 \%$.

Experiment 2: The speed of image pattern recognition using different algorithms. To guarantee a smooth user experience, the recognition algorithms should be fast enough to provide a real-time experience. We tested the training dataset collected using different feature extraction algorithms to measure the performance. Although most of the speed are similar to each other, the FAST is on average faster than the rest.

In this paper, challenges during the development of this project and sample design structure will be discussed in detail in Section 2. Experiments implemented to examine the validity of this program will be discussed in section 4. Published works similar to or related to this project will be discussed in section 5. Finally, concluding remarks as well as future perspectives will be discussed in section 6 .

\section{Challenges}

Building a mobile application for the first time with a new programming language is not an easy task. Throughout the course of the development, we ran into several challenges that needed overcoming. Here is a brief overview of some of the most difficult challenges that we faced when developing this app.

\subsection{Challenge 1}

Initially we intended to use existing biology models on the market. However, after research there's not sufficient models that fit my education purpose of demonstration to students. For example, the neuron structures we found on sketchfab are relatively accurate however complex models, that different parts like axons and dendrites are hard to identify for students [18]. To solve this, we decided to build my own 3D models. We decided to build the models in blender, 
since blender is a free and open-source graphic software that gives users a lot of freedom and flexibility to model anything they want. While crafting the 3D models, we realized I need to craft a 3D model by adjusting each point, edge, and face. This limits the complexity of my models since we did not have matured 3D modeling skills. Therefore, we re-chose the biological molecules for modeling.

\subsection{Challenge 2}

There are also issues in constructing 3D models themselves [19]. For example, originally, we intended to use two cylinders and a UV sphere to construct each single phospholipid to create a phospholipid bilayer model for the action potential demonstration, but there is a gap in the connection of the cylinder and the UV sphere. After we deleted one face of the UV sphere, the open-space is a polygon instead of a circle, which the cylinder doesn't fit perfectly into. Therefore, we changed the construction method to that like a tree branch. I extended one face of the UV spear to make it protrude out, and we adjusted the protruded part to make it look like the phospholipid tail.

\subsection{Challenge 3}

There were lots of problems during the coding process as well. At first, the objects did not turn at the desired speed, so different parameters were tried for $\mathrm{x}, \mathrm{y}$, and $\mathrm{z}$ to get the preferable turning. When testing the capture function, the 3D model appeared but it did not show its full feature. To solve this issue, camera position and the image to model ratio was adjusted. This allowed the model to appear right at the place of the image and be the appropriate size that can rotate properly and show the full feature of the models. These are just examples of the changes and improvements, and in my mind one most interesting aspect of constructing an application is constantly refining it.

\section{Solution}

\subsection{Augmented Reality Model}

We used Vuforia Engine to create model target of biological molecules. In model target, we do not need to scan the real object instead we use computer-aided design (CAD). Using 3D data makes the recognition and tracking of the object more powerful and faster. To create the model target we require Unity 3D, which is a Vuforia Model target generator, physical object, matching 3D CAD model and Vuforia Engine.

There are some characteristics that we need to consider when we create our 3D biological molecule objects to augment and optimize our AR model for better performance. First, the objects must have sufficient geometric details and the object must be rigid. Also, we reduce our 3D model down just to the part that the camera can identify to recognize the object.

To make our augmented reality application, we used Blender, Unity and Vuforia. (See figure 1) First, we created 3D model of Biological molecules by using Blender; In Blender, we used UV wrapping process to imitate the texture and material of biological molecules. Then we created our Vuforia database that contains target images (biological molecules). In this database we upload the image of biological molecules in png and jpg format.[9] After we created the image target and 3D models, we imported the target images and the 3D models to Unity, so the 3D models can be mapped with the target images. [10][11] 


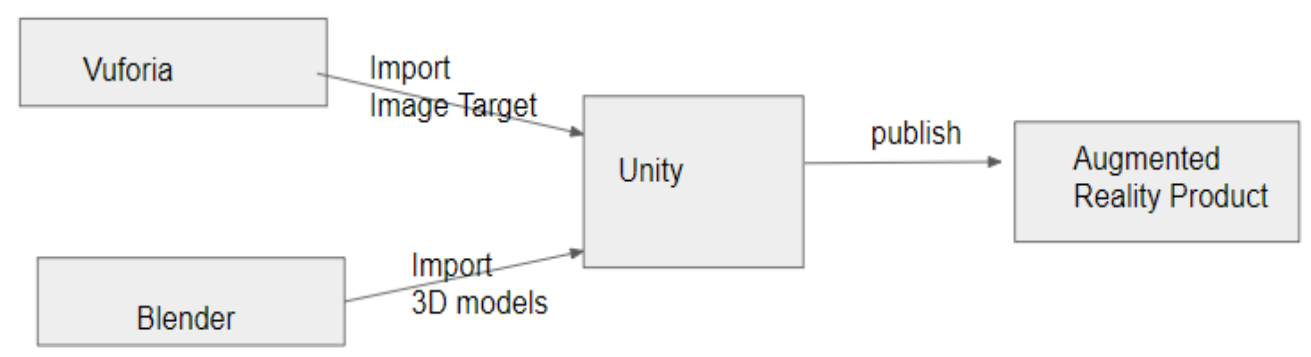

Figure 1: Components of Augmented Reality Model.

\subsection{Computer Vision Mobile Phone}

We used computer vision library Vuforia to recognize the 2D image. Vuforia provides diverse feature detectors such as Scalar-Invariant Feature Transform (SIFT) and Speeded up Robust Features (SURF) [16]. In order to get the most accurate approach, we analysed 3 different detection algorithms and compared their result. The approaches that we have chosen are Features from Accelerated Segment Test (FAST), Difference of Gaussians, and Determinant of Hessian. FAST is a corner detection algorithm that focused on efficiency [15]. Difference of Gaussians is an algorithm that involves the subtraction between 2 Gaussian kernels with different standard deviations [17].

\subsection{Mobile Phone Application}

Our solution is a program built based on Unity that can scan and recognize specific pictures and demonstrate a 3D model along with explanation of the corresponding image. This application can be used in different platform such as Android, iOS and Windows. This application contains 3 different levels of depths. (see Figure 2) First there is a menu page, with three buttons "capture", "collection", and back. When a user clicks on the capture button, the application enables the camera of the phone, so the application can recognize the specific 2D biological molecule image. After the specific image is recognized, the page is updated with the biological molecule information and its corresponding 3D model of the image. For example, in figure 3, we can see in the right side the 3D Brain image on top of the 2D image of the Brain. The 3D model is pre-built, and images are preloaded into the database. I scanned some images from the Campbell AP Bio textbook chapter 48(Neurons, synapses, and signaling), and built corresponding models.[2] 


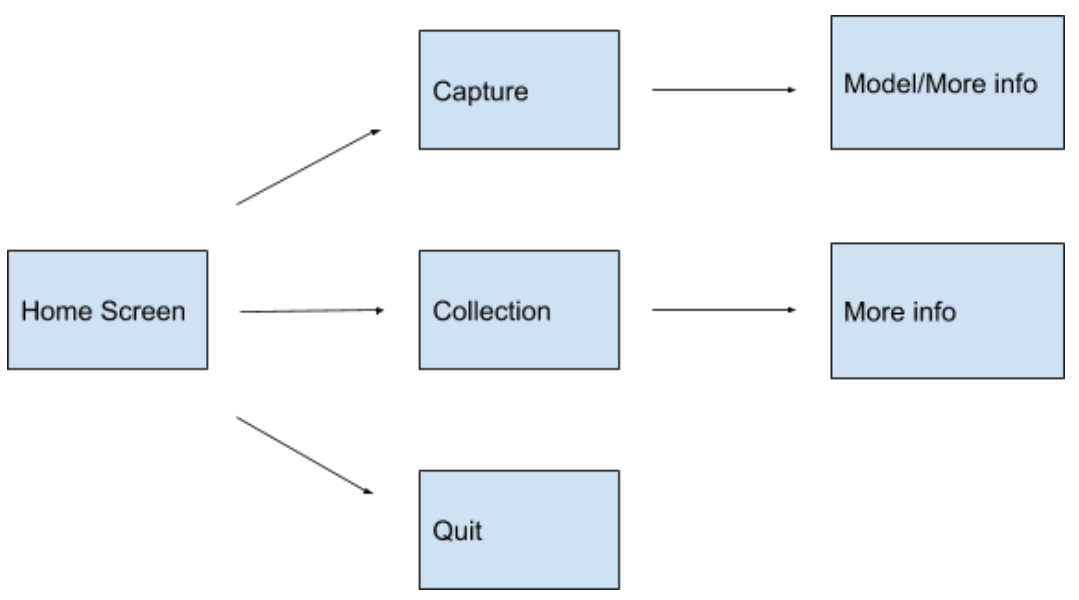

Figure 2: Overview of 3D Biological molecule image User Interface
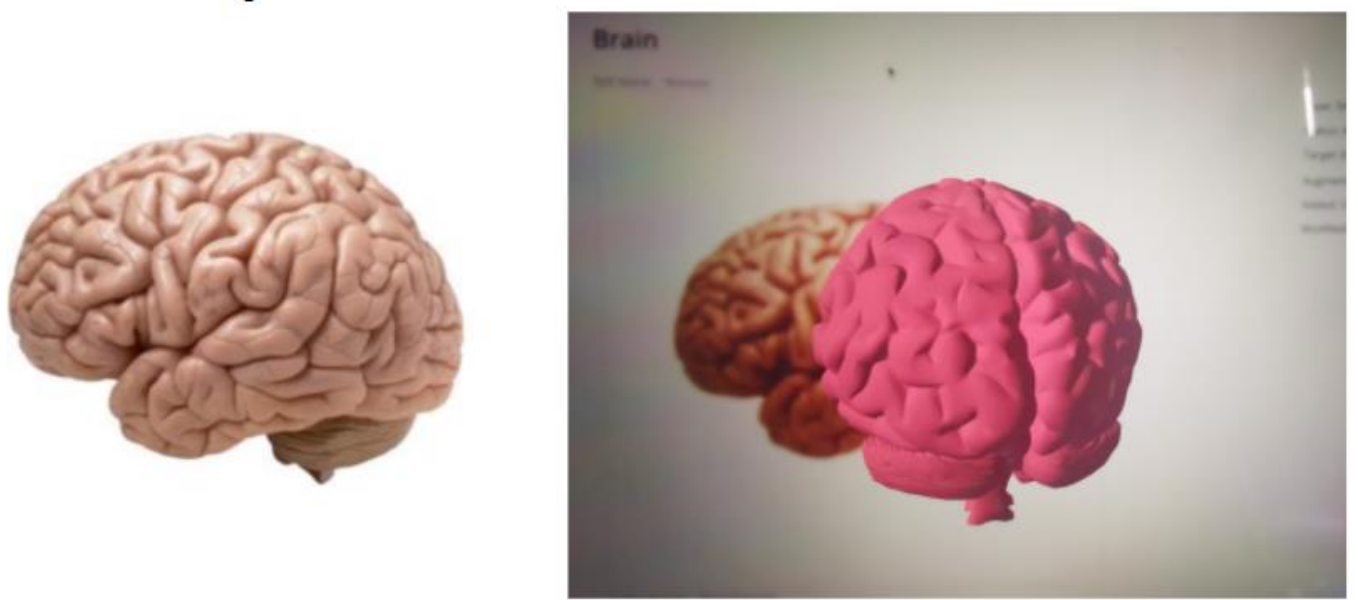

Figure 3: 2D image of a brain image from a book(left). 3D image of a brain using Augmented - reality (right)

Apart from scanning, users can also see the collection when clicked on the "collection" button, where there is a list of buttons users can scroll through to select the model they want to view. (See Figure 4) When clicked on the specific model, the page is directed to the 3D model page. In Figure 5, we can see that after the user clicked in one of the categories, the information of the chosen biological molecule is showed on the screen with its specific image. On the left and right, we can observe protein channel and neuron information with its corresponding image, respectively. 


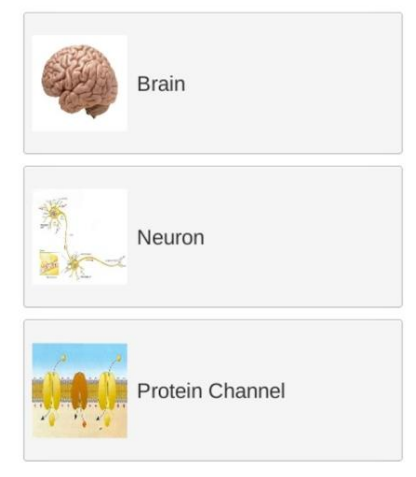

Figure 4: Collection Menu
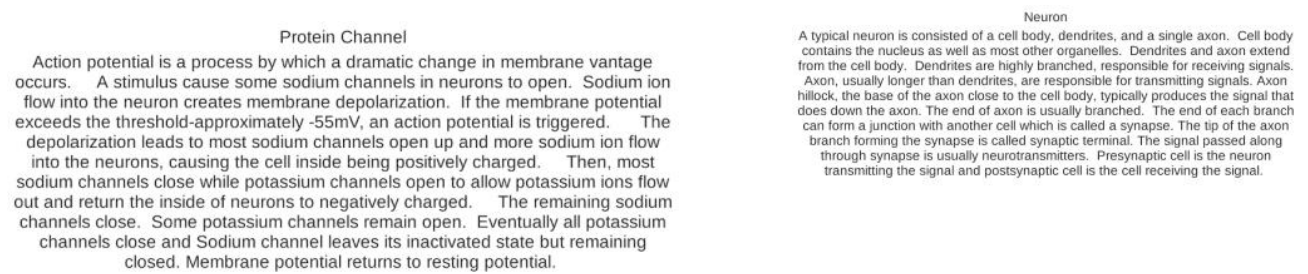

out and return the inside of neurons to negatively charged. The remaining sodium

channels close and Sodium channel leaves its inactivated state but remaining

closed. Membrane potential returns to resting potential.
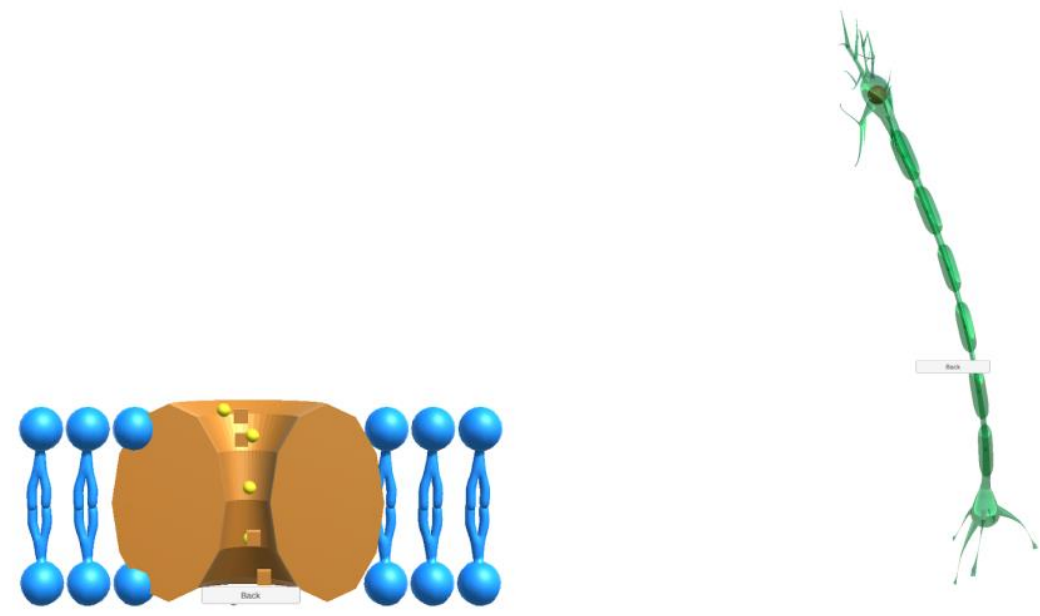

Figure 5: More Information Screen. Protein Channel (Left). Neuron (right).

\section{EXPERIMENT}

Two experiments have been conducted to measure the performance of the system.

\subsection{The accuracy of the image pattern recognition}

The core experience and performance of the AR application is based on the accuracy of the image pattern recognition based on the view captured by the camera. We have tested the accuracy of the image pattern recognition using different algorithms. The core image pattern recognition algorithm relies on the image feature extraction methods. Different feature extraction algorithms to recognize the image pattern based on the chosen sampled test images are experimented, such as FAST, Difference of Gaussians, and Determinant of Hessian. It turns out that FAST has the highest accuracy of $94.5 \%$ as shown in the chart below. 


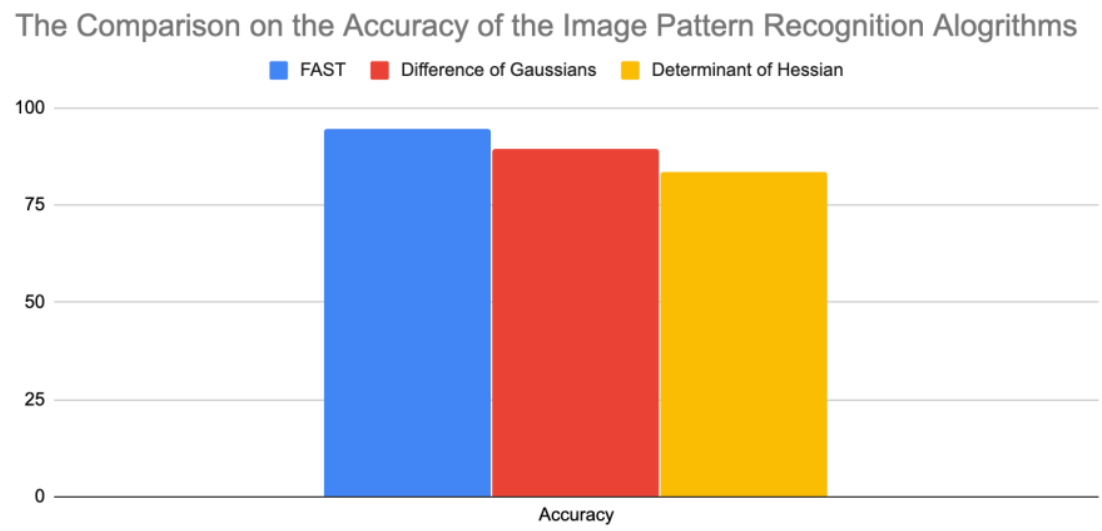

\subsection{The speed of the image pattern recognition}

The speed of image pattern recognition using different algorithms. In order to guarantee a smooth user experience, the recognition algorithms should be fast enough to provide a real-time experience. We tested the training dataset collected using different feature extraction algorithms to measure the performance. Although most of the speed are like each other, the FAST is on average faster than the rest as shown in the Figure below.

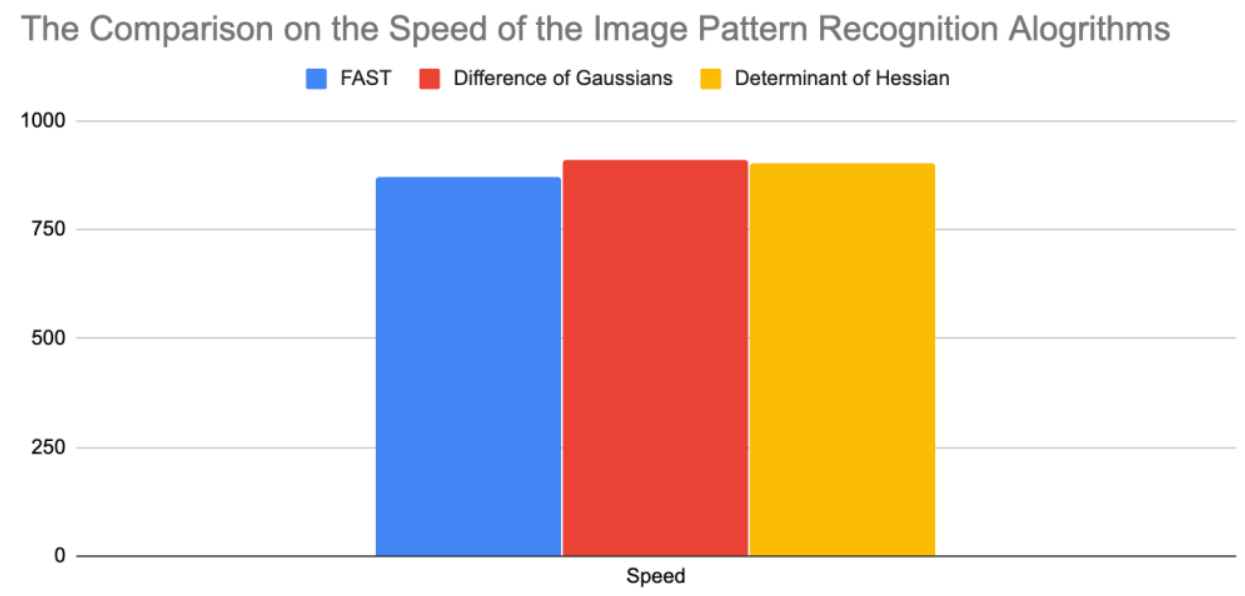

\section{RELATED WORK}

Frantz, T. et all addressed the data tracking issue of Microsoft's HoloLens, a tool in neuronavigation, by using Vuforia and Unity[1]. They demonstrated that HoloLens with Vuforia achieves better result and hologram stability than using it without Vuforia. In this work, we used Vuforia to recognize biological molecule images and Unity to create our application.

Amin, D. and Govilkar, S. presented a comparative study of Augmented Reality SDK's such as Metaio SDK, Vuforia SDK, Wikitude SDK, D'Fusion SDK and ARToolKit SDK. [3] They compared them based on license type, platform supported, marker generation, tracking and overlaying capability. Even though Vuforia has some disadvantages such as it does not provide utility function that can load 3D model from diverse formats, we decided to use it since it can support different platforms (Android, iOS and Windows). 
Takala T. et all implemented a 3D modeling with Blender.[6] In their study, professional and novice 3D artists used their program to create 3D models. Even though the application was easy and fun to use, it has posing accuracy issues. In our work, we used Blender since it works very well with Vuforia and Unity.

Liu X. et all described how to use Vuforia and Unity to design a game.[8] They explained how to set up Environment Development and Production Process of AR Tower Defense Game. First, the explained step by step of how to establish the environment. Then the step that you must follow to Process of AR Tower Defense Game Generation. Finally, how to generate the game. In their application they can play video, set animation, and interact by using virtual button mode.

\section{CONCLUSION AND FUTURE WORK}

Learning biological molecules can be very difficult and challenge for learners since they need to try to visualize the biological molecules so they can understand their functionality. They can only use 2D images that are provided in Biology books or Sketchfab and Turbosquid - platforms that buy, sell, share and publish 3D content model- and 3D physical biological molecule models. However, these methods are inefficient and costly. In order to solve this issue, we designed an Augmented-Reality application for Biological molecules that students can use to learn and study biological molecules. Our application has a collection mode in which users can access to a database that contains Biological molecules. In this database, there are information of biological molecules and their corresponding picture. Another option is the capture mode in which learners it to visualize a biological molecule in 3D. This capture mode uses the camera to recognize the 2D image of a biological molecule as input and output the 3D image and information of the target image.

To develop, the AR application, we used Vuforia, Blender and Unity. We decided to use Vuforia, an AR software, since it supports iOS, Android and Window platforms, which were our target platforms. Also, we decided to use Blender, a 3D modeling, and Unity, a game engine, to show the visualization of the biological molecules and they work very well with Vuforia.

We used 3 different algorithms: FAST, Difference of Gaussians, and Determinant of Hessian for the image pattern recognition. Our experiments show that FAST algorithm is more effective and efficient since it is faster than other algorithm and provides highest accuracy of $94.5 \%$.

There are some limitations like the range data in Vuforia database and 3D models are limited. As result, we plan to construct more 3D models for a wider range of biology molecules and integrate the models into the software in the next version. In addition, we plan to add a feature that create animation effect and labels for the 3D models, so that learners can understand and study biological molecules.

\section{REFERENCES}

[1] Frantz, Taylor, et al. "Augmenting Microsoft's HoloLens with vuforia tracking for neuronavigation." Healthcare technology letters 5.5 (2018): 221-225.

[2] Urry, Lisa A, Michael L. Cain, Steven A. Wasserman, Peter V. Minorsky, and Jane B. Reece. Campbell Biology. New York: Pearson, 2017. Print.

[3] Amin, Dhiraj, and Sharvari Govilkar. "Comparative study of augmented reality SDKs." International Journal on Computational Science \& Applications 5.1 (2015): 11-26.

[4] Linowes, Jonathan, and Krystian Babilinski. Augmented Reality for Developers: Build practical augmented reality applications with Unity, ARCore, ARKit, and Vuforia. Packt Publishing Ltd, 2017. 
[5] Flavell, Lance. Beginning blender: open source 3d modeling, animation, and game design. Apress, 2011.

[6] Takala, Tuukka M., Meeri Mäkäräinen, and Perttu Hämäläinen. "Immersive 3D modeling with Blender and off-the-shelf hardware." 2013 IEEE Symposium on 3D User Interfaces (3DUI). IEEE, 2013.

[7] Hess, Roland. The essential Blender: guide to 3D creation with the open source suite Blender. No Starch Press, 2007.

[8] Liu11, Xinqi, Young-Ho Sohn, and Dong-Won Park. "Application Development with Augmented Reality Technique using Unity 3D and Vuforia." International Journal of Applied Engineering Research 13.21 (2018): 15068-15071.

[9] Peng, Fuguo, and Jing Zhai. "A mobile augmented reality system for exhibition hall based on Vuforia." 2017 2nd International Conference on Image, Vision and Computing (ICIVC). IEEE, 2017.

[10] Glover, Jesse. Unity 2018 augmented reality projects: build four immersive and fun AR applications using ARKit, ARCore, and Vuforia. Packt Publishing Ltd, 2018.

[11] Borycki, Dawid. Programming for Mixed Reality with Windows 10, Unity, Vuforia, and UrhoSharp. Microsoft Press, 2018.

[12] McCue, T. J. "How to find cool things to 3D print: Sketchfab, MyMiniFactory, Thingiverse." (2019).

[13] Squid, Turbo. "3D Models, Plugins, Textures, and more at Turbo Squid."

[14] Vuforia, Vuforia - enable your apps to see, https://www.vuforia.com/

[15] Gao, Qing Hong, et al. "A stable and accurate marker-less augmented reality registration method." 2017 International Conference on Cyberworlds (CW). IEEE, 2017.

[16] Andreasson, Karl Johan. "Stabilizing Augmented Reality views through object detection." (2017).

[17] Lv, Yaqi, et al. "Difference of Gaussian statistical features based blind image quality assessment: A deep learning approach." 2015 IEEE International Conference on Image Processing (ICIP). IEEE, 2015.

[18] Msanjurj, PatrickZhiaran, Préfontaine, A., Uspalenko, V., Zoilo, Budi, . . . Mumladze, N. (n.d.). Publish \&amp; find 3D models online. Retrieved September 09, 2020, from https://sketchfab.com/

[19] Bartonek, Dalibor, and Michal Buday. "Problems of Creation and Usage of 3D Model of Structures and Theirs Possible Solution." Symmetry 12.1 (2020): 181.

[20] Cooper, A. Kat, and M. T. Oliver-Hoyo. "Creating 3D physical models to probe student understanding of macromolecular structure." Biochemistry and Molecular Biology Education 45.6 (2017): 491-500.

(C) 2020 By AIRCC Publishing Corporation. This article is published under the Creative Commons Attribution (CC BY) license. 